\title{
IOI 2015 Report
}

\author{
Artem IGLIKOV, Mansur KUTYBAYEV, Bakhyt MATKARIMOV \\ Nazarbayev University \\ 53 Kabanbay batyr ave., Astana 010000, Kazakhstan \\ e-mail: \{artem.iglikov, mansur165, bakhyt.matkarimov\}@gmail.com
}

\begin{abstract}
The International Olympiad in Informatics (IOI) is an annual international informatics competition for individual students at schools for secondary education from various invited countries, accompanied by social and cultural programmes. We present a report on the 27th International Olympiad in Informatics, July 26 - August 2, 2015, Almaty, Kazakhstan (IOI'15), organized by the Ministry of Education and Science of the Republic of Kazakhstan, Republican Scientific and Practical center "Daryn", al-Farabi Kazakh National University and supported by Mayor of Almaty and Mayor of Almaty region. IOI' 15 established a new IOI record with 322 contestants from 83 countries, participated in IOI' 15 and awarded by 161 medals ( 27 gold, 55 silver and 79 bronze), Jeehak Yoon from the Republic of Korea is absolute winner of IOI'15. At IOI'15 Java was first time introduced as IOI official programming language. In this report we pointed attention on issues happen as well as things that done well.
\end{abstract}

Keywords: IOI, programming contest, International event organization and management.

\section{Introduction}

IOI is one of the world's top level Olympiads for secondary schools students, among International Mathematical (since 1959) / Physics (since 1967) / Chemistry (since 1968) / Biology (since 1990) Olympiads. Initiated by UNESCO and starting from 1989 in Pravetz, Bulgaria, IOI constantly develops, especially in the level of scientific and technical solutions. The IOI's official site is http://ioinformatics.org, for general information on IOI we refer readers to the website and the following IOI documents: IOI Regulation $^{1}$, IOI syllabus ${ }^{2}$ and the ITC/ITWG guidelines ${ }^{3}$.

The President of the Republic of Kazakhstan, Dr. Nursultan Nazarbayev, made order in 1996 on governmental support and development of secondary schools for gifted students, and in 1998 the Government of the Republic of Kazakhstan established a new state enterprise, Republican Scientific and Practical center "Daryn" with primary goal to discover, encourage and give recognition to gifted students by developing and supporting

\footnotetext{
${ }^{1}$ http://ioinformatics.org/rules/index.shtml

${ }^{2}$ http://www.ioinformatics.org/a_d_m/isc/iscdocuments/ioi-syllabus.pdf

${ }^{3}$ http://wiki.ioinformatics.org/wiki/HostingAnIOI
} 
special educational programs and activities. Kazakhstan hosted 36th International Mendeleev Chemistry Olympiad in 2002, Almaty city. Kazakhstan subregion of the Northeastern European Regional Contest of the ACM International Collegiate Programming Contest was created in 2003. From 2004 in Almaty city was organized annual International Zhautykov Olympiad on Mathematics, Physics and Computer Science (IZhO, n.d.), hosted by the Zautykov Republican Specialized Physics-Mathematics Secondary Boarding School for Gifted Students. Kazakhstan hosted 7th Asian Physics Olympiad in 2006, Almaty, and 51st International Mathematical Olympiad in 2010, Almaty-Kokshetau-Astana. Based on these achievements, Kazakhstan applied to IOI Executive Director as IOI potential host in 2010. At IOI'10 in Waterloo, Canada, Republic of Kazakhstan was selected by IOI International Committee as IOI'15 future host. Before IOI'15 Kazakhstan hosted 46th International Mendeleev Chemistry Olympiad in 2012, Astana city, http://mendeleev . kz/ and 45th International Physics Olympiad in 2014, Astana city http://ipho2014.kz/.

\section{IOI Host Committees}

Steering Committee: Aslan Sarinzhipov, Minister of Education and Science, Chairman; Yessengazy Imangaliyev, Vice-Minister of Education and Science, Vice-Chairman; Galymkair Mutanov, Rector of the Al-Farabi Kazakh National University; Sholpan Kirabayeva, Director of the Republican Scientific and Practical center" Daryn”, Secretary; Akhmetzhan Yessimov, Mayor of Almaty city; Yerbolat Dossayev, Minister of National Economy; Tamara Duissenova, Minister of Health Care; Kalmukhanbet Kassymov, Minister of Internal Affairs; Asset Issekeshev, Minister of Investment and Development; Bakhyt Sultanov, Minister of Finance; Erlan Idrissov, Minister of Foreign Affairs.

Host Scientific and Technical Committee: Bakhyt Matkarimov, Adilet Zhaksybai, Sergazy Kalmyrzayev, Ulugbek Adilbekov, Myrzakerei Miras - Nazarbayev University; Darkhan Akhmed-Zaki, Zhanl Mamykova, Natalya Surina, Erbolat Kalaman, Shyngyz Rabat, Pavel Chekanov, Askar Akshabayev - Al-Farabi Kazakh National University; Artem Iglikov, Azizkhan Almakhan, Madyar Aitbayev, Nurlan Zhussupov, Askar Aitzhan, Yesskendir Sultanov, Bektur Suleimenov - Kazakh-British Technical University; Mansur Kutybaev - International Information Technologies University; Fuad Hajiyev - ADA University, Azerbaijan; Georgiy Korneev, Nikolay Vedernikov, Gennady Korotkevich - ITMO University, Russia; Egor Kulikov, Elena Andreeva - Moscow State University, Russian Federation; Michael Mirzayanov - Saratov State University, Russia; Alexander Klenin - Far-Eastern State University, Russia; Ali-Amir Aldan - Massachusetts Institute of Technology, USA.

$50+$ staff specialists from involved organizations works full time during IOI'15 week. $150+$ volunteers selected from active members of the host University volunteers club (team guides, organization staff) and from former participants of IOI and other Olympiads (HSTC volunteers).

IOI'15 was fully supported by the Government of the Republic of Kazakhstan. About 2000K was reserved/allocated for IOI'15 from the Ministry of Education and Science of the Republic of Kazakhstan, $\$ 1556 \mathrm{~K}$ was spent for the main event, including purchase 
of facilities $\$ 652 \mathrm{~K}$ and $\$ 97 \mathrm{~K}$ for IT Infrastructure works/HSTC/Technical staff. In total, guest fee was $\$ 93 \mathrm{~K}$.

\section{Preparatory and IOI Present Host Actions}

IOI'15 website created in 2011 and general information with photos about Kazakhstan was first time distributed at IOI'11, Pattaya, Thailand. Initially IOI'15 was planned in the capital Astana, in 2014 Almaty city and al-Farabi Kazakh National University were announced as the host of IOI'15. Programming contest for 300+ onsite contestants and $10000+$ submits for evaluation with full feedback is a hard task, and IOI'15 was a first case in Kazakhstan. Previous to IOI Kazakhstan experience in programming contests organization was reported in (Iglikov et al., 2013). The following preparatory actions was crucial: 1) using IOI CMS (Mares and Blackham, 2012; Maggiolo et al., 2014) at national and international programming contests in Kazakhstan, Artem Iglikov recognized as IOI CMS developer; 2) organization of Asian-Pacific Informatics Olympiad APIO'14, hosted by Kazakh-British Technical University, Almaty (APIO’14, 2014); 3) hosting all-Russia team Olympiad in programming for secondary schools in Almaty at K.Satpayev Kazakh National Technical University ${ }^{4}$. Programming language Java accepted as official programming language in various programming contests, including ACM ICPC. Martin Mares, ISC/ITWG, evaluated Java solutions for IOI'13 tasks, created by Egor Kulikov, Pavel Mavrin and others, and reported to ISC at IOI'14, Taipei, Taiwan. ISC recommended Java as official IOI'15 programming language. In 2014 Egor Kulikov accepted IOI'15 HSTC member invitation. In 2014-2015 IOI CMS development team added full support for Java.

Communications with IOI countries representatives was by e-mail and private Google group "IOI 2015 Team Leaders". List of country contact emails extracted from the IOI Registration system, managed by Eljakim Schrijvers https://ioiregistration.org/. All foreign participants, including IOI committees and Host scientific and technical committee (HSTC), were registered in IOI Registration system. IOI'15 invitation letters was generated through IOI registration system, as well as various reports, e.g. list of participants with relevant information, including email, meals preferences, travel data, etc. For visa support procedures Host created forms and instructions, published on website and distributed by email two month before IOI, visa support procedures takes up to 30 days. Free of charge landing visa at Almaty International airport was organized for all teams who completed required procedures.

Call for tasks was made in December 2014 with submission deadline at January 31, 2015. Contestants machines specifications and operation system image was published in June 2015. Significant changes in IOI'15 competition rules from previous IOI was Java as official programming language, allowing multi-threaded programs at the contest time, and limits on competition print job size. 3 practice session tasks published 2 weeks before IOI'15. IOI'15 and 9th IOI conference programmes was published at the host website in July.

\footnotetext{
${ }^{4}$ http://neerc.ifmo.ru/school/russia-team/index.html
} 


\section{IOI Committees Meeting}

IOI IC/ISC committees meeting organized in Almaty at February 26 - March 2, 2015. All IC/ISC members participated this event, for newly created ITC committee Host decided to organize meeting few days before IOI. IC inspected IOI' 15 venue, making many objective suggestions with short checklist. IC minutes was published at IOI official web site. 43 unique tasks submitted for IOI'15, including 6 submissions from HSTC. HSTC rejected 11 submissions, due to various reasons, and 25 tasks shortlisted for selection by ISC. Finally, ISC selects 9 tasks for IOI'15, including 3 backup tasks: Scales, Eryk Kopczyński, Poland; Teams, Adam Karczmarz, Poland; Boxes with souvenirs, Monika Steinova, Slovakia; Towns, Bang Ye Wu, Taiwan (IOI'14 backup task); Sorting, Weidong Hu, China; Horses, Mansur Kutybayev, Kazakhstan; Liar, Ulugbek Adilbekov and Sergazy Kalmurzayev, Kazakhstan (IOI'15 backup task, opened at IZhO'16 (IZhO, n.d.)). Cultural programme of IOI committees meeting includes opera presentation Tosca and visit of Shymbulak mountain area.

\section{IOI Organization}

IOI'15 venue formed by nearly located al-Farabi Kazakh National University campus with newly built Student hotel (contestants), Atakent Park Hotel (team leaders) and RitzCarlton Hotel (IOI committees, tasks authors, invited guests). Walking time between team leaders and contestant's hotels is about 25 minutes. Student hotel was opened free of charge without meals for early arrived teams contestants from July 23. For IOI'15 needs host University allocated al-Farabi library building, Director Kalima Tuenbayeva. Team's registration was at al-Farabi library. Opening/Closing ceremonies performed in the U.Dzholdasbekov Palace of Students. Most of activities organized in Atakent Park Hotel for team leaders, and in host University campus for contestants. IOI Doctor (medical) room allocated in al-Farabi library and Student hotel, as well as host University medical center was ready to serve for IOI. At IOI'15 3 medical treatment cases registered. 10 air conditioning and climate control facilities was installed in competition hall (6) and Student hotel (4). We reserved at least two rooms/halls for any IOI activity, except single competition hall. IOI committees rooms, IOI office, translation session hall, GA meeting hall was allocated both in Atakent Park Hotel and al-Farabi library. Rooms in Student hotel was not identical, e.g. for 2 or 4 persons, mirrors was only on 1st floor, etc. Rooms for girls was allocated on the 1st floor, mixing contestants from different countries, also we allocated contestants rooms starting from 1st floor based on IOI total medals rank of countries. In hotels for adult participants most of rooms was single, and we sorted participants by age to allocate rooms. Student cafeteria at host University campus was allocated for contestants and team guides. For leaders breakfast was on a residence, lunch and dinner was at Atakent Park Hotel. Lunches at contest days was organized for all participants at host University campus. Farewell party was at Atakent Park Hotel. Transportation was organized within IOI venue and for all excursions, for guest excursions two big busses was allocated. There was a shortage of minibuses, and 
planned hourly shuttle at IOI venue was not implemented. Life stream translation was not at IOI'15, daily video distributed with YouTube by Azhar Rakhletova, easily found by search "IOI 2015", also video/photo materials published at IOI'15 website. IOI'15 schedule was traditional 8 days for IOI.

\section{Cultural Programme}

IOI'15 cultural programme includes two whole day excursions for all participants, two whole day and 3 Almaty city excursions for guests, various sport/entertainment events and invited lectures. Whole day guest excursions were to the Big Alma-Arasan gorge with Nursery "Sunkar", and Kazakh aul "Huns". Almaty city excursions were to the Central State Museum of Kazakhstan, «Kok Tobe» mountain park with panoramic view of Almaty city, Park of 28 Guardians of Panfilov's division, Saint Ascension Cathedral, Ykhylas Museum of Folk Musical Instruments. Day 4 excursion was to the high-mountain sports complex "Medeu", including visit of Shymbulak glacier at $3000+\mathrm{m}$ above the sea level; and Kazakh State Circus 45 years anniversary presentation. Unfortunately, day 6 Turgen gorge mountains area excursion was canceled due to official emergency notification on flood flow, not happen actually, and excursion was limited to acquaintance with National customs and traditions.

IOI'15 Host organizing committee thanks to Chris Peterson from Massachusetts Institute of Technology for lecture "How to apply to MIT (and other USA colleges)" at July 30 with various presents, e.g. books signed by MIT professors, including "Introduction to Algorithms" by Thomas H. Cormen, Charles E. Leiserson, Ronald L. Rivest and Clifford Stein. Kazakhstan governmental program Bolashak supports education abroad and our students are very interested in presentations of this kind. We did not consider this presentation as a promotion of MIT, in fact we also invited lecturers from few other Universities and ICPC community, and only Chris Peterson comes to IOI'15. It would be better to make open announcement before IOI for similar activities.

\section{9th IOI Conference}

IOI conference traditionally organized in two days during contest time in parallel with Question/ Answers sessions at July 28-30, 2015. IOI conference program published at host website and includes 15 presentations with workshop on IOI CMS contest management system, moderated by Stefano Maggiolo, ITS member. Special session of IOI conference was organized for Kazakhstan teachers.

\section{Quarantine}

During Quarantine time contestants allowed to enter Student hotel garden and IOI volunteers organized Dance club / Entertainment programme. 


\section{Translation Sessions}

Translation sessions organized at Atakent Park Hotel. For translation we asked team leaders to bring their own laptops, and less than 10 laptops were requested from organizers. At IOI'15 was used IOI Translation system created in Taiwan for IOI'14 with minor modifications. At the first translation session a large printing queue was caused by inefficiently organized printing procedures with operator intervention, this was improved for the second session. In the evening before contest day 2 a power outage happen in the hotel, causing one hour delay in GA meeting start time. Fortunately, both backup and central power recovered by power service engineers on duty, and we did not switch to the backup plan - translation hall at host University without coffee break. Translation nights continued up to 5 a.m. In total, 454 task statements with notices was prepared during translation sessions and published at IOI'15 host and IOI official websites.

\section{Question/Answer Sessions}

Question/Answer sessions organized at Atakent Park Hotel in parallel with IOI Conference. At IOI'15 clarification forms from contestants was scanned and distributed within "IOI 2015 Team Leaders" Google group, being visible to all team leaders, and text translation from any team leader who knows question language was accepted. Most of contestant's questions in fact were handled within IOI CMS, e.g. questions written in English or any other language, known by ISC members. Only six questions from contestants were handled at Question/Answer sessions.

\section{Contest Tasks}

For IOI tasks preparation we used automated platform for creating programming contest problems Polygon ${ }^{5}$, developed at Saratov State University, Russia, by Michael Mirzayanov team from 2008. Polygon automates contest tasks preparation, organizing effective team work on tasks and preventing typical errors, and supporting user access management, version control, issue-tracking, integration with popular test systems, contest tasks archive compilation, long-time online backups, tasks search and classification capabilities. For security reasons separate instance of Polygon was installed by Michael Mirzayanov on our servers with two levels of authorization.

Boxes with souvenirs. Number of subtasks: 6. First two subtasks are easy and require basic knowledge of programming with simple logic. Third subtask could be solved by "brute force" algorithm. Starting from fourth subtask, contestants have to make simple observations about the structure of the problem. Fourth subtask has a lot of different solutions; the most popular one could be dynamic programming with 2 states. Subtask 5 and 6 could be solved only with most important observation of the task. The difference is in implementation details of the algorithm which could give better performance. Tests

\footnotetext{
${ }^{5}$ https://polygon. codeforces. com
} 
development for this problem was relatively easy. Problem has special tests for checking correctness of solutions on important observations.

Scales. Number of subtasks: 1. This problems has no subtasks. Score for this problem depends on effectiveness of the algorithm. We used all the available tests for this problem, which could be easily generated and grouped by subtests. 56 different scores was on this task at IOI'15 competition.

Teams. Number of subtasks: 4. First subtask could be easily solved by "brute force" algorithm. The second subtask needs knowledge of well-known sorting algorithms. Third and fourth subtasks need advanced geometry and data structure algorithms. A lot of tests were prepared for this problem, including all corner cases. Most of tests were prepared to test programs on time limits, which was crucial for this problem.

Horses. Number of subtasks: 5. First subtask very easy one. Starting from second subtask contestants should make observation about algorithm structure. Second one is just easy implementation of this algorithm. Third subtask needs another crucial observation, which helps to solve this subtask. Difference between third and second subtask is only in constraints. The last subtasks could be solved only with advanced data structures and programming techniques. Tests for this problem were quite tricky, because it consist of a huge amount of corner cases. And there is a lot of space to make some mistake in program. There are a lot of different tests that covers huge amount of occasions.

Sorting. Number of subtasks: 6 . This problem has a lot of different subtasks, because it could be solved in many different ways. Of course not all of them are efficient enough. Most of the subtasks have some unique constraints which makes the problem easier than the problem itself. First three subtasks don't require main observation to solve the whole problem. Difference between these subtasks is implementation difficulty. For the next three one needs observation about the problem structure to be successfully solved. Each time contestant needs to make more effort using additional technique or implement additional data structure. Problem needed only some restricted amount of tests, which was prepared using small test generators. They cover almost all cases, including corner cases, small cases and large testcases.

Towns. Number of subtasks: 6 . This problem may be divided into more than 6 subtasks, it was decided to fix the number of subtasks on 6. Each subtask could be treated as a different problem, because each of them has some specific constraint on some parameters. Depending on the contestant observations there might be slightly different algorithm to solve with different score. The problem was divided by most interesting cases to solve. Tests preparation for this problem was hardest one in the contest, consisted from the algorithm which is hard to test with fixed amount of tests, because of the randomized solutions. Jury tried to prepare a lot of different tests that increase probability of failure of wrong solutions. Most of them were prepared depending on wrong solutions written by jury itself and by beta testers.

When all task statements was completed we did not write tests verification programs, based on final constraints and separated from tests verificator, already created by tasks developers, this caused formally invalid test cases. Initially prepared graders was not enough secured to prevent attacks. IOI tasks analysis with test data published at IOI'15 host and IOI official websites. 


\section{IOI System}

IOI system needs low feedback time and high availability/fault tolerance and high performance/ throughput networking evaluation system, built on relatively low cost facilities. All parts of IOI'15 system was reserved, $10 \%$ of laptops, $100+\%$ of servers, $100 \%$ network core switches and trunk lines, and $10 \%$ of network switches and network cables to end-point devices. IOI' 15 facilities were purchased in 2015 by open bid according to the laws of the Republic of Kazakhstan. Many kinds of facilities were provided by supporting organizations. IOI'15 facilities includes 1) uninterruptible power supplies: built-in with laptops, 20 new 2kVA UPS for every network switch, 1 new Fujitsu APC Online $20 \mathrm{kVA}$ in server room, external 800kVA mobile power generator for competition building; 2) various facilities for installation of power network in competition hall; 3) 415 HP ProBook 450 G2 laptops with external keyboard, mouse, mousepad as contestants machines/grading system workers; 4) 30+ external monitors provided for contestants by request; 5) servers: 4 new HP/ProLiant DL380Gen9, 6 new (6+) Fujitsu blade servers; 6) 1 new Fujitsu NetApp backup storage system; 7) 14 Alcatel-Lucent 48 port layer-2 network switches, supporting VLANs and 1 GigE Ethernet; 8) 4 Alcatel-Lucent layer-3 10 GigE switches at network concentration points; 9) Fujitsu rack; 10) network cables, testers, cable channels, telecommunication boxes, etc. for network installation; 11) 5 new fast color printers; 12) 5 new (+5) fast black-white printers; 13) 3 new (+7) big Samsung monitors as information desks, network monitor, contest results online presentation, etc.; 14) 5+ projectors for presentations; 15) 10 new climate control facilities installed, 6 in competition hall, 4 in student hotel; 16) 1 audio system for announcements in competition hall; 17) various video/photo translation/publication facilities for public media coverage; 18) 6 mobile communications jammers for quarantine; 19) 1 voting system for GA meetings.

ITC/HSTC report follows: Laptops was chosen to have more modern technical characteristics than at previous IOI, and to maximize work time on battery, e.g. 15.6" display and Core i5 CPU, and to fit in the budget. 336 laptops were allocated for contest hall, 60 for grading system, 19 for translation session and other needs. Host received similar (but not exact) laptops about 3 months before IOI, to prepare the system and test battery work time. The laptops arrived about 1 month before IOI. The network was not yet set up, so we couldn't fully test the laptops at that time, we just checked that every laptop can successfully be turned on and boots the pre-installed system. The primary servers used for the contest were HP DL380 Gen9. Everything was installed on one server. Another server was a full copy of the main and had database was being replicated to it, so in case of problems we could switch to it in minutes (and nothing would be lost). Third server was used for live backups of contestant machines; we couldn't do this on the first server due to high load on the file system. Fourth server was used for translation sessions. We also had blade servers and tower machines ready to use as a backup system. We used 10 Gbps network interfaces on the server with $10 \mathrm{Gbps}$ switches in the server room. Switches in the contest hall all had 1 Gbps links to contestant's laptops and 210 Gbps uplinks to the server room. All switches outside of the contest hall were backed up, so if any one 
fails network would be still up. We had several spare switches for the contest hall and we could configure them in few minutes to replace any failed switch. All switches were managed remotely by university network administrator. Cable network built safely using dual camera cable raceways for both power and digital networks. Competition hall has 6 video cameras to monitor from ISC/ITC rooms.

Contestant sample images preparation: once we had the IOI'15 laptops, we took 5 of them for setting up the contestant software. Initial software setup was performed using Ubuntu package-management software (APT). Basic software installation was scripted (INSERT REPO LINK). Some manual configuration had to be done for setting up help in some IDEs (INSERT MANUAL LINK). Also, several days before the IOI we updated the image. Two versions of Eclipse 3.8 and 4.4 were provided. The 3.x branch considered to have better performance, while the 4.x branch has more features. After the installation of software following issues was detected 1) KWrite could not open any file for reading (fixed by installing missing KDE packages); 2) Default destination of Java API for Eclipse, NetBeans and IntelliJ IDEA points to Oracle internet site (JDK links was fixed in configuration); 3) Default $\mathrm{C}++$ help files are 15 years old (replaced by actual StdLibC++ help files); 4) StdLibC++ help files are not complete (additional help files was download from http://en.cppreference.com/); 5) Free Pascal IDE has no help files (help files was downloaded and installed); 6) Free Pascal IDE fails on debugging complex programs (not fixed); 6) Code::Blocks hangs when multiple instances started in short period of time (not fixed). For each Editor/IDE following requirements was checked: 1) It is possible to write, build, run and debug a programs solving "Search" practice session problem on all supported languages (a lot of minor misconfigurations found and fixed); 2) Help files are available for all supported languages (required help files was downloaded and configured); 3) It is possible to save/load files (failed by KWrite); 4) Printing are supported (printing is not implemented in Sublime Text editor and have bugs in Code::Blocks, to alleviate later issue contestants was instructed to use "Print to PDF" or use different IDE/Text editor for printing). Keylogging software (logkeys) was installed on contestants' workstations. It allows monitoring contestant activity before and during the contest. Some of the contestants performed activity on theirs workstations before start of the contest (most of them - unintentionally, like sleeping on keyboard) and was warned about that. The side-effect of the key logging was ability to determine times when a contestant computer was hang. It became possible due to timestamps that accompanies pressed keys and clear message of key logger restart. Therefore, it is possible to determine the time of last key press before the hang-up and first key press after it. So we have a good upper bound on hand-up time.

Imaging contestant machines: for imaging we follow Bernard Blackham report for IOI 2013 (Blackham, 2013). We have set up a dhcp boot server (dnsmasq), which forwarded laptops to boot TinyCoreLinux from tftp server (atftpd). The imager script was being downloaded after TCL is booted, so we didn't have to update TCL image each time we change the imager script. The imager script wipes the partition table, re-partitions the drive, and starts udp-receiver to receive the main partition image. We were able to re-image up to 90 machines with the speed about $900 \mathrm{Mbps}$, which for $10 \mathrm{~GB}$ image took about 1-2 minutes. We tried to re-image all the machines at once, but this didn't 
work well (probably required some nicer setup). We decided not to lose time on this and reimaged the hall by 3-4 rows (each row was up to 25 machines). Even in this case it took about 10-15 minutes to reimage everything. We also didn't bother on TCL image size and contestant image size and had no issues with it. During imaging the addressing was the following: all laptops in the VLAN 18 with addresses 10.18.row.place / 16. Server had one interface in VLAN 18 with address 10.18.0.1 / 16. During the contest the addressing was the following: each laptop in different VLAN $(1600+($ row -1$) * 100+$ place -1$)$ and address 10.row.place. 2 / 24. Server had over 300 virtual interfaces with addresses 10.row.place. 1 / 24.

All managing of contestant's machines and workers was done remotely (after several tries). We could: turn on laptops remotely with wake-on-lan (which saved us from a lot of walking), selectively re-image laptops, start memtest or badblocks on all the laptops, do anything we want on the laptops during the imaging script is running (thanks to Bernard for embedding a backdoor), do anything we want on the laptops after imaging with SSH (laptop contained servers public SSH key) or NetAdmin. To control and monitor contestant workstations we used Java-based NetAdmin tool by Georgiy Korneev. It monitors current state of each computer in the network several times per second and allows issuing control command to all workstations or selected part of them. There is a command queue for each workstation, so the command executes only when workstation is accessible and all previous commands has been finished successfully. NetAdmin allows issuing of server-side (local) and workstation-side commands (remote). Serverside command executed on server by NetAdmin itself. For example, Copy contestant data command looks as follows: scp $-r$ day\} $\{\mathrm{ip}\}: /$ home/ contestant, where \{day\} and $\{i p\}$ are placeholders for current contest day (day0, day1, or day2) and IPaddress of the computer to copy data to. By default, command executes on all computers simultaneously, but this is not the case for Copy Contestant Data command - if you try to copy $100 \mathrm{M}$ simultaneously on 360 computers this will result in terrible network performance. NetAdmin is able to throttle execution of such kind of commands to specified number of simultaneous executions. Workstations-side (remote) commands are executed by special remote execution service, installed on controlled workstations. Both remote execution service and NetAdmin server authorizes each other using TLS certificates. Another option is to use ssh command on server side. An example of client-side command is Reboot: reboot $-\mathrm{fn}$. Notice, that there is no need to specify exact computer to reboot, as long as the command is run on that specific computer. NetAdmin support offline commands that executes event when workstations are not accessible. Offline commands are useful for wake-on-lan and similar scenarios. NetAdmin also has been used to monitor and control grading system invocation workstations.

During the competition, we performed backups of contestant home directory on each workstation. On days 1 and 2 backups scheduled to run each three minutes. During the practice session backups was performed each minute to test network throughput and to measure influence on contestants. Average backup of all contestant workstations took 40-50 seconds, with peaks up to 60 seconds. Backups was performed using rsync incremental backups (--link-dest), this allows to make snapshots of home directory, while preserving hard drive space and network throughput. Using this scheme the initial 
backup of the single workstation has size of $300+\mathrm{MB}$. To reduce this size we implemented cross-workstation incremental backups. In this scheme we took the full backup of only one «original» workstations (still 300+MB), while backups of the other workstations was made as incremental relative to «original». This allows us to reduce the size of the initial backup to $5 \mathrm{MB} /$ workstation. Most of this $5 \mathrm{MB}$ are files regenerated by Gnome/Unity after detection of new hardware ids after reimaging. New backup scheme allows us to dramatically reduce time and space required for the initial backup. Unfortunately, the backup time of all workstations was 5-7 mins, even when there are no actual changes was made. Profiling shown than workstations CPU and network usage was almost negligible, while performance was capped by server hard disc. Further investigation shown, that most of the time is spend by rsync to create hard link to each unmodified file during backup. To alleviate this issue we switched from Ext4 to BTRFS that gave us 5 times boost. To speed up backups even more, we decided not to backup large amount of files that should (almost) never change or are regenerated on startup. This two tweaks combined reduced backup time of all workstations to $40-50 \mathrm{sec}$, while decreasing workstation and network load. Test backup restore was performed several times, and no issue was found. Unfortunately, backup restore during day 1 analysis resulted in hang of heavily used workstations. After reboot all workstations become ok. Further investigations shown that hangs was due simultaneous update of Gnome configuration files and cache by both user and rsync. There are two scenarios to work around this issue: 1) Perform backup restore only when workstation is not in use. This is the main scenario in the case of unrecoverable contestant workstations failure. In this case, restore took about $30 \mathrm{sec}$, which is small compared to time to try recovering workstation, and moving contestant to another one; 2) Restore backup to different directory. This scenario was used on day 2 result discussions, when backups were restored to dayX/backup directories.

We used master version of CMS (which has evolved greatly during the year before IOI'15) with some modifications. Main improvement of this year was testing a submission in parallel on all the workers on different test cases. So if there are some idle workers, contestant would receive the result much faster than if it would be tested on one worker as it was before. This lead to an issue with ES performance, but it was solved by Stefano and Bernard before day 2. Proper patches for CMS will be posted in the official repository. Other CMS modifications included: 1) specifying subtasks inclusion in dataset options (when subtask 2 can include all the test cases from subtask 1 according to the task statement, that could be specified in the dataset options, and results of evaluation on test cases of subtask 1 will be included into results of evaluation on test cases of subtask $2 ; 2$ ) displaying results was done in an aggregated form (for each appeared evaluation outcome we showed number of test cases and maximal used memory and time); 3) Oracle JDK support; 4) many improvements for Polygon importer. There were several problems with Java support: multi-threading, memory limit, time limit. Oracle JDK is multithreaded by nature. During testing we observed about 17 threads required to just start the program for Oracle JDK 8 on Ubuntu 15.10 x64. We had several choices: 1) limit number of threads with the sandbox (this would be problematic, because JVM can start GC any moment on a separate thread); 2) limit the number of threads the contestant program is allowed to start (this should work with GC which is not being started 
by contestant program, but there was a theoretical possibility that standard libraries used by the contestant could start some threads); 3) allow multi-threading. We have chosen to allow multi-threading and counted the execution time as the sum of execution times of all threads of the program. This lead to an interesting issue: if the program used amount of memory close to the JVM heap size, then JVM would start GC on a separate thread, and its working time will be added to the total execution time. To overcome this issue we raised memory limits and JVM heap size (both -Xmx and -Xms). Another problem is that standard data input classes (Scanner, BufferedReader) in Java are quite slow. With help of Egor Kulikov we re-wrote all graders to use the raw FileInputStream, buffering and parsing the input in the grader itself. After this, surprisingly, Java input become much faster than input on $\mathrm{C}++$. So we had to rewrite $\mathrm{C}++$ input procedures as well (reading number of bytes from the input and parsing them manually). After that the same technique was applied to FreePascal graders.

\section{Competition}

Day 1 and 2 contests were started at 9:00 as planned by schedule. IOI'15 CMS evaluation statistics: 5762 solutions at competition day 1 evaluated in total on 325118 tests, 8845 solutions at competition day 2 evaluated in total on 470563 tests. In total 371 print jobs from 114 contestants was executed, 9 pages was a maximum per single job. Submits statistics by tasks vs. programming languages with number of different contestants and submits presented in Table 1. C++ used by most of contestants, and Java usage approaches $\mathrm{C} /$ Pascal usage. Java perspectives may be only estimated locking usage dynamics on next IOI's.

ISC/ITC/HSTC reports on issues happen at contest time: Hardware setup, including servers, workstations and networking was very solid. There was no major issues was found, while several minor issues was fixed: 1) glitches on workstation screen when the lid is moving ( 2 contestant, workstations were replaced); 2) heavy glitches for few

Table 1

Programming languages usage at IOI'15

\begin{tabular}{llccccccr}
\hline & C++ & & Java & & Pascal & \multicolumn{3}{c}{ C } \\
\hline day1 & $\mathbf{3 1 2}$ & $\mathbf{5 2 7 8}$ & $\mathbf{5}$ & $\mathbf{9 0}$ & $\mathbf{5}$ & $\mathbf{8 1}$ & $\mathbf{4}$ & $\mathbf{5 8}$ \\
\hline boxes & 308 & 2380 & 5 & 42 & 5 & 27 & 3 & 33 \\
scales & 281 & 1503 & 4 & 19 & 4 & 23 & 4 & 15 \\
teams & 263 & 1395 & 5 & 29 & 4 & 31 & 3 & 10 \\
\hline day2 & $\mathbf{3 1 2}$ & $\mathbf{8 1 0 7}$ & $\mathbf{1 1}$ & $\mathbf{1 4 8}$ & $\mathbf{5}$ & $\mathbf{1 7 9}$ & $\mathbf{4}$ & $\mathbf{1 1 6}$ \\
\hline horses & 305 & 3339 & 11 & 61 & 5 & 70 & 3 & 22 \\
sorting & 304 & 3006 & 6 & 66 & 4 & 72 & 4 & 93 \\
towns & 177 & 1762 & 4 & 21 & 2 & 37 & 1 & 1 \\
\hline
\end{tabular}


seconds when video mode is changed (probably bug in video card drivers, does not affect contestants); 3) two contestants was provided with additional monitors (by request); 4) no mouse pads on practice session (mouse pads were provided by request on days 1 and $2 ; 5$ ) workstation hang-ups (real origin is undetermined, 8 contestants was affected during day 1 , and 4 during day 2).

\section{Day 1:}

At the beginning one of 12 CMS servers was not working properly due to misconfiguration. As a result electronic statements and grading was unavailable for roughly 1/12 of all contestants. They could still read the hard copies. This was reported around 9:15 and fixed within $\sim 5$ minutes.

Around 9:40, a cheating case was detected. In problem Scales a contestant managed to read the internal data of the grader. We fixed the grader, so that this type of hacking would be much more difficult. When doing that we faced a CMS bug, which resulted in grading not working for $\sim 20$ minutes. After that, the outstanding solutions were graded quickly.

At 10:30 we discovered a problem in test data for task Teams. A task statement condition was not satisfied in one test case, 2 students got affected. The first one was the one who reported the issue, so he added a workaround within few minutes. The second student submitted a solution that solved the incorrect test 13 minutes later after his incorrectly graded submission. Some other students already passed this test. A rejudge was on the way, but we realized that it would not finish before the end of the contest. Because of that, around 13:40 we have announced the details of the problem.

Around 12:30 the same CMS bug has shown up. Before it got fixed, around 12:33 we had a power problem with UPS on network switches, which caused the CMS server to be unavailable for everybody. It was fixed in about 10 minutes. During this time, the contestants could work on their computers. After few minutes the CMS bug was "fixed" (graders were restarted). Unfortunately, one contestant has to reboot his computer during power failure period. This reboot takes a lot of time, since DHCP server was inaccessible at this moment. It is recommended to pin dynamic IP addresses in future installations.

At 12:55 the allowed interval between submissions increased from 1 to 5 minutes (per task). This was dropped down to 1 for the last 8 minutes of the contest. During the second half of the contest the grading time was around 18 minutes. To the best of our knowledge, all grading results were reported within 25 minutes.

Several computers locked up during the contest and they had to be rebooted. We have analyzed the logs, but the reason remains mysterious (we are still investigating). In one case, it happened during the network outage, so the machine did not come up immediately. The student has lost about 5 minutes, so we decided to extend the contest by 5 minutes for him. However, additional analysis of log files revealed that some other machines could have been unusable for up to 13 minutes. They were rebooted by the volunteers, who did neither tell us nor recorded the details, and the contestants did not complain, so we cannot be sure how serious the problem was. The volunteers will receive better instructions for day 2 .

A large number of clarification requests were received due to students not knowing how to compile their program in their preferred environment (mostly Code::Blocks). 
They had included graderlib.c and grader.cpp into their project, which caused multiple symbols to be defined.

Public clarification actions: 1) In the beginning: Aman can give multiple souvenirs when he is in a section; 2) Graders read I/O from file; 3) Scales grader behavior for incorrect queries; 4) Late: sample grader prints not only the sequence, but also the number of queries; 5) Late: Teams may give bad results.

8 machines froze and got rebooted incidents of rebooting from 3 to 15 minutes.

Questions received: 1) lots of technical questions - we assisted the students; 2) questions regarding the rules - we gave the full answered; 3) task related questions - often answered ANSWERED IN TASK DESCRIPTION or INVALID QUESTIONS. Exceptions: questions regarding something that was clarified - then we tried to provide a very helpful answer. Question/Answer translations worked fine.

Analysis of day 1 issues: 1) some cases of freezing computers may have been caused by Code::Blocks grabbing the keyboard and mouse and then locking. However, there may have been other causes, too; 2) There were 4 students affected by the regrade on Teams. To gather more information on sporadic hang-ups of contestant's workstations, the remote syslog facility was set up. Syslog analysis allows attributing some of hangups to Code::Blocks and finding the way to alleviate them. To test performance of remote syslog workstations was simultaneously rebooted several times. No missing log messages or substantial network load was detected during reboots.

\section{Day 2:}

During the day 2 all announcements were dubbed on the screen as popups using notify-send.

There was a small issue in the sample grader for Towns. This was updated in CMS before the contest and pushed to the machines at 09:05. 09:11: E-1 report could not extract zip file, confirmed, using local versions for sorting at 09:15. Several issues rose about day 2 graders, first at 09:13. There were two versions of graders; both of them were the same. 09:32 clarifications notice that horse.out is 0/0. The .out files are results of running sample graders, announcements made at 09:46. 09:20 ranking issue: submissions with ids from day 2 overwrote submissions from day 1. Fixed at 09:40. 11:40 Found and fixed a typo in the announcement sorting.1.in $\rightarrow$ sorting1.in. 13:07 another ranking issue: some scores became lower after refresh. Fixed in 2 minutes. This was likely caused by a faulty restart of one the ranking servers.

During the contest day 2, one more issue was found: the bug in Code::Blocks that caused UI to hand while debugging some programs. Two affected contestants were instructed to use pkill in such circumstances. Recorded issues at day 2: 1) L-5 hardware 12:36-12:41; 2) G-15 multiple Code::Blocks freezes ( 15 min total lost) caused by the contestant repeatedly trying the same thing; 3) J-10: 12:30-12:35; 4) I-13: 13:40-13:44 Code::Blocks.

ITC investigated CMS issues and reported: After the ISC confirmed the problem on a test case for Teams, the ITC inserted new test dataset in CMS and started the background judging. The system handling the queue of submissions saw an increase in workload that exposed an application level deadlock causing frequent freezes. Therefore the background judging proceeded slowly, and eventually it was decided to switch to the 
new dataset before its judging caught up. As the rate of submission increased, the deadlock was triggering again, requesting manual intervention each time. As judging became slower, contestants increased their submission rate as they could not wait for the (potentially positive) previous results. This forced us to increase the minimum time between submissions to 5 minutes. The cause of the deadlock was found and fixed by Bernard between day 1 and day 2. In parallel to this, one reason for the higher than expected workload was CMS change to distribute each test case to a single worker, which has the advantage of giving much lower latency when the system is relatively free. The unexpected drawback of this change, in a setting with high number of workers such as the IOI, was that the system, handling the queue, was overwhelmed by the communication with the workers. This problem was patched by Stefano between day 1 and day 2 (where each evaluation was split in about half a dozen packets rather than one per test case).

\section{Award Ceremony}

322 IOI'15 contestants awarded 161 medals, 27 gold (rounded up), 55 silver (rounded up) and 79 bronze. Jeehak Yoon from the Republic of Korea is single absolute winner of IOI'15 with perfect score 600 from 600, he was awarded IOI trophy. IOI Distinguished award was to Don Piele, USA, post mortem. Special presents were given to girls IOI'15 contestants (Kazakhstan tradition).

\section{Conclusion}

In IOI'15 participated 83 Official Teams ( 77 teams with 4 contestants, 3 teams with 3 contestants, 2 teams with 2 contestants, 1 team with 1 contestant), 0 Observing Countries, 1 President, 1 ED, 10 IC, 8 ISC, 7 ITC, 24 HSTC; 322 Official Contestants, 161 Leaders/ Deputy Leaders, 62 Guests +7 Invited Guests +2 children. Hosting IOI is an exceptional event in a lifetime experience. Thank you very much IOI community for giving us the opportunity to show a small part of our beautiful country! Welcome Kazakhstan again!

\section{Acknowledgements}

We greatly appreciate efforts and hard work of more than 200 specialists to organize IOI'15, including staff, volunteers, engineers from the Ministry of Education and Science of the Republic of Kazakhstan, Republican Scientific and Practical center "Daryn", al-Farabi Kazakh National University, administration of Almaty and Almaty region, ITMO, Saratov SU, Moscow SU, Far-Eastern State University, ADA University, MIT, Kazakh-British Technical University, International Information Technologies University, Kazakhstan Hewlett-Packard office, ALSI, Albeta Kazakhstan, Emergency service Almaty, Kazakh State Circus, Medeo \& Shymbulak administration, Kazakhtelecom and many others. Thank you very much! 


\section{References}

IZhO (n.d.). International Zhautykov Olympiad. http://izho.kz/

Iglikov, A., Gamezardashvili, Z., Matkarimov, B. (2013). International Olympiads in Informatics in Kazakhstan. Olympiads in Informatics, 7, 153-162.

Mares, M., Blackham, B. (2012). Introducing CMS: a contest management system. Olympiads in Informatics, 6, 86-99.

Maggiolo, S., Mascellani, G., Wehrstedt, L. (2014). CMS: a growing grading system. Olympiads in Informatics, $8,123-131$.

APIO'14 (2014). Asian-Pacific Informatics Olympiad 2014. http: //olympiads .kz/apio2014/

Blackham, B. (2013). Bernard Blackham report on IOI'13.

http://www. ioinformatics.org/locations/ioi13/

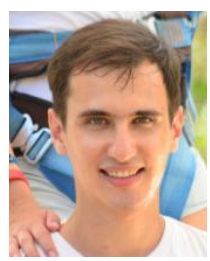

A.V. Iglikov - ISC member, Taiwan 2014, ITC member, Host Country 2015. Technical director of Kazakhstan subregion of the Northeastern European Regional Contest of the ACM International Collegiate Programming Contest. Jury chairman of the Kazakhstan National Olympiad in Informatics, International Zhautykov Olympiad (on Computer Science).

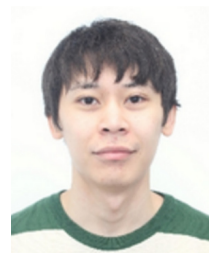

M.U. Kutybayev - IOI Team leader of Kazakhstan 2014. ISC member, Host Country 2015. Author of task "Horses" (IOI 2015). Jury chairman of the Kazakhstan National Olympiad in Informatics, International Zhautykov Olympiad (on Computer Science).

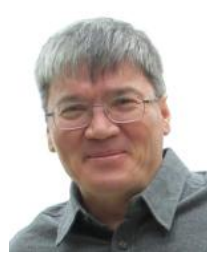

B.T. Matkarimov - IOI Team leader of Kazakhstan from 2005. IC member, Host Country 2013-2016. Chair of IOI 2015. Initiator and Jury chairman of Kazakhstan subregion of the Northeastern European Regional Contest of the ACM International Collegiate Programming Contest. Jury chairman of the Kazakhstan National Olympiad in Informatics, International Zhautykov Olympiad (on Computer Science). 